\title{
The Neuroprotective Effects of Xenon on Neonatal Rats with White Matter Damage by Regulating Expression of microRNA-210 and HIF-1aThe Neuroprotective Effects of Xenon on Neonatal Rats with White Matter Damage by Regulating Expression of microRNA-210 and HIF-1a
}

\section{Xiangyun Yin}

The Affiliated Hospital of Qingdao University

Jixiu Zhao

Jining First

Jian Jiang

The Affilaited Hospital of Qingdao University

Hongmin Xi

The Affiliated Hospital of Qingdao University

Xianghong Li

The Affiliated Hospital of Qingdao University

\section{Lulu Zhang}

The Affiliated Hoapital of Qingdao University

Hong Jiang ( $\nabla$ jianghong@qdu.edu.cn )

Qingdao University Medical College

Research

Keywords: preterm, Xenon, microRNA-210,HIF-1a, white matter damage

Posted Date: August 18th, 2020

DOl: https://doi.org/10.21203/rs.3.rs-60259/v1

License: @ (i) This work is licensed under a Creative Commons Attribution 4.0 International License. Read Full License 


\section{Abstract}

Background:Premature infant is a significant health care burden. White matter damage (WMD) is a leading cause of acute mortality and chronic morbidity in preterm. Xenon (Xe) intervention was given to the 3-day-old neonatal rats with brain white matter injury. By detecting the changes in the expression level of microRNA210 and hypoxia inducible factor $1 a(\mathrm{HIF}-1 \mathrm{a})$ in brain tissue before and after xenon intervention, we can research the molecular basis and the mechanism of neuroprotective on effect of xenon on brain white matter damage in neonatal rats.

Methods:Three-day-old SD rats were randomly divided into sham group(Group A, $n=24$ ), lipopolysaccharide(LPS)+hypoxia-ischemia(HI) group (Group B, n=24) and LPS+HI+Xe group ( $n=72)$. The onset of $X e$ inhalation started at 0,2 and 5 hours in subgroups $C, D$,and $E$ respectively. We investigated the neurobehavioral deficits by performing TUNEL and hematoxylin and eosin (HE) staining and examining the expression of miR-210and HIF-1a in brain tissues via RT-PCR and western blot.

Results: Xe treatment improved the histological alterations and decreased the number of apoptotic cells in group $C$ pups.Compared to group A,Detection of miR-210 level by RT-PCR. the expression level of miR210 in neonatal rats' periventricular tissue increased significantly at all time points in group $B$ $(p<0.05)$.While the expression level of miR-210 in brain tissues of group B was significantly lower at $48 \mathrm{~h}$ and $72 \mathrm{~h}$ than that of group $\mathrm{C}(\mathrm{p}<0.05)$.Similarly,Detection of HIF-1 a protein by Western blot. The level of HIF-1a protein in group B brain tissues was significantly higher than that of group $A$ at each time point $(p<0.05)$, Xe treatment resulted in a marked increase in HIF-1 a in C,D, and $E$ subgroups $(P<0.05$, compared to group B).

Conclusions: These results demonstrate that the expression of HIF-1a and miR-210 increased in periventricular tissues and Xe could relieve the white matter damage by up-regulating the expression of HIF-1a and its target gene miR-210.The Xe therapeutic time window was within 5 hours after intervention, the sooner the better.

\section{Background}

WMD is a prominent neurological deficit observed in preterm birth. WMD is a leading cause of acute mortality and chronic morbidity. [1] Miller $S P$. previously reported that more than $25 \%$ of premature infants with brain injury may have different degrees of neurological sequelae,and the incidence of cerebral palsy is as high as 10\%.[2] HI and inflammation are two main pathogenic mechanisms of brain injury in premature infants.[3,4] But there is no effective treatment for WMD at present. It is very important to explore a safe and effective intervention.

Xe is a noble gas used in general anesthesia.[5] Studies have shown that xenon has protective effects on ischemia-reperfusion injury of brain.[6,7] Moreover, xenon exhibited neuroprotective activity for the treatment of ischemic brain injury in combination with mild hypothermia in animal models.[8-11] Xenon is known to cross the blood-brain barrier (BBB) and has a low blood/gas solubility, which will reduce the 
risk of developing adverse effects.[12-14]Previous reports demonstrated that the anesthetic effect of xenon was primarily mediated through the inhibition of N-methyl-D-aspartate (NMDA). White matter injury has become the main cause of nervous system dysplasia in premature infants, but there is no effective treatment for WMD at present. It is very important to explore a safe and effective intervention. $[15,16]$ Particularly, Ma et al demonstrated that xenon could induce the activation of hypoxia-inducible HIF-1a and provide preconditioning effects on ischemic renal injury in mice.[17]However, the exact molecular mechanism of xenon-mediated neuroprotection remains to be elucidated. Moreover, the neuroprotective effect of xenon in WMD has not been adequately investigated.

MicroRNAs (miRNAs) are endogenous, non-coding small RNA molecules of about 22 nucleotides in length and are involved in regulating gene expression, primarily at the posttranscriptional level. MiRNAs play a ubiquitous role in many vital biological processes, such as cell differentiation, proliferation, and apoptosis,etc. miR-210 is recognized as one of the most stable and significant microRNAs, and it is a stable target gene of HIF-1 a,during hypoxia,HIF-1a is up-regulated,which induces the expression of microRNA-210 to increase.[18]In this study, we investigated the neuroprotective effects of xenon in a neonatal rodent model of WMD. Further, we examined the impact of xenon on the outcome through measuring the expression of HIF-1a and miR-210 in neonatal brains.

\section{Methods}

\section{Animals and Experimental groups}

Pregnant SD dams were obtained from the Experimental Animal Center of Qingdao, China. A total of 120 postnatal days $(P) 3$ rat pups, weighing 7.5-11.3 g, were housed in temperature- and humidity-controlled cages with their moms in a 12-hour light/dark cycles. The experimental protocol was revised and approved by the Institutional Animal Care and Use Committee of Qingdao University. All measures were taken to minimize animal discomfort.

Pups were randomly assigned into 3 groups: sham group (Group $A, n=24), L P S+H I$ group (Group $B, n=$ $24)$, and LPS $+H I+X e(n=72)$. According to the onset of xenon treatment, the LPS $+H I+X e$ group was further divided into three subgroups, Group $C(n=24)$, $\operatorname{GroupD}(n=24)$ and Group $E(n=24)$.

\section{Induction of WMD and Xenon treatment}

As we have done previously[19] ,Pups were either administered lipopolysaccharide (LPS, $0.05 \mathrm{mg} / \mathrm{kg}$, Escherichia coli 0111:B4; Sigma, USA) or normal saline (NS, Sham group) via intraperitoneal (i.p) injection. To avoid LPS-induced body temperature changes, pups were housed with their mothers after LPS or NS injection in an incubator for 3 hours to maintain their body temperature at 33 to $34{ }^{\circ} \mathrm{C}$ before HI.[20] Next, pups were anesthetized with $5 \%$ chloral hydrate $(0.01 \mathrm{ml} / \mathrm{gm}$ body weight, i.p) and HI was induced as described previously.[21] Briefly, the right common carotid artery (CCA) was exposed and separated from nerves and veins. Subsequently, the CCA was permanently ligated with $4-0$ surgical silk, the wound was sutured and pups were returned to their cages to recover from anesthesia. The entire 
surgical procedure never exceeded 5 minutes. Sham-operated pups underwent the same operative procedure except for the ligation of CCA. After 1 hour recovery, pups in Groups B and C were placed in an airtight $3 \mathrm{~L}$ container partially submerged in a $36^{\circ} \mathrm{C}$ water bath, and exposed to humidified $8 \%$ oxygen(a mixture of $8 \% \mathrm{O}_{2}$ and $92 \% \mathrm{~N}_{2}$ ) with a flow rate of $3 \mathrm{~L} /$ minute for 1 hour. Following hypoxia, pups in Group $B$ were returned to the cage until they were sacrificed. Animals in group C, D and E were placed in a separate closed circuit container with 50\% xenon (mixed with 30\% oxygen and $20 \%$ nitrogen) for 3 hours.Following $\mathrm{HI}$, the onset of the xenon treatment was delayed for 0,2 hours, and 5 hours in groups $\mathrm{C}, \mathrm{D}$ and $\mathrm{E}$, respectively. Carbon dioxide was removed from the container by soda lime pellets. Pups were decapitated at $0,24,48$ and 72 hours following xenon treatment, and control pups from groups $A$ and $B$ were simultaneously decapitated.

\section{Hematoxylin and eosin staining}

Brains were excised as described previously.[19] Briefly, pups were anesthetized with chloral hydrate and fixed on an operating panel. Next, the heart was exposed and a $10 \mathrm{ml}$ syringe with $4 \%$ paraformaldehyde was inserted from the left ventricle to the aorta. Paraformaldehyde was injected into the heart until clear liquid flowed out from the atrium and the extremities became pale and stiff. Subsequently, the skull was systematically stripped to expose cerebral tissue. The periventricular brain tissue was dissected, immediately placed in $4 \%$ paraformaldehyde solution at room temperature for 48 hours, dehydrated in ethanol series, and embedded in paraffin. Paraffin blocks were coronally sectioned at a 10- $\mu \mathrm{m}$ thickness from the genu of the corpus callosum to the end of the dorsal hippocampus. Hematoxylin and eosin (HE) staining was performed in four sections per brain. Stained sections were observed under a light microscope (OLYMPUS BX41, Olympus, Center Valley, PA,USA).

\section{TUNEL staining}

The apoptotic cells were detected with terminal deoxynucleotidyl Transferase Biotin-dUTP Nick End Labeling assay(TUNEL,Roche) according to the instructions. 10 visual fields were randomly selected to count the number of positive cells, and the average value was obtained under a light microscope (OLYMPUS BX41, Olympus, Center Valley, PA, USA).

\section{Western blot analysis}

Brains were quickly extracted and the periventricular tissues were dissected and stored in aliquots at $-80^{\circ} \mathrm{C}$ until further analysis. Periventricular brain tissues were homogenized in a cold lysis buffer supplemented with protease inhibitors and protein concentrations were determined using a BCA protein assay kit (Elabscience, Wuhan, China). Protein samples $(40 \mu \mathrm{g})$ were separated using $10 \%$ SDS-PAGE and blotted onto polyvinylidene fluoride membranes overnight at $4{ }^{\circ} \mathrm{C}$. Membranes were incubated with the appropriate primary antibody including anti-HIF-1a (1:500; Elabscience, Wuhan,China) and anti-GAPDH (1:1,000; Elabscience, Wuhan, China) overnight at $4{ }^{\circ} \mathrm{C}$. Next, membranes were incubated with anti-rabbit IgG antibodies conjugated to horseradish peroxidase (HRP; 1:50,000; Elabscience, Wuhan, China)and proteins were visualized by enhanced chemiluminescence. Band signals were quantified using imaging software (BandScan 5.0). 


\section{Real-Time Reverse Transcription-PCR}

Total RNA was extracted from brain tissue using Trizol (Aidlab, Lot:252250AX, Beijing, China) followed by quantification and reverse transcription using HiScript Reverse Transcriptase (RNase H) reagent Kit (Gene Copoeia,MD, USA) following the standard protocols. Next, real-time PCR was performed using the $2 \times$ Allin-one tmqPCR MixKit(Vazyme Biotech, Nanjing, China). Then using specific miR-210 and an endogenous control U6 stem-loop primer, miR-210 or U6 stem-loop reverse transcriptase primers in a $20 \mu \mathrm{l}$ system buffered with RT buffer and Distilled De-ionized Water(ddH2O). The RT thermal cycle program was as follows: $25^{\circ} \mathrm{C}$ for $5 \mathrm{~min}, 50^{\circ} \mathrm{C}$ for $15 \mathrm{~min}, 85^{\circ} \mathrm{C}$ for $5 \mathrm{~min}$ and $4^{\circ} \mathrm{C} 10 \mathrm{~min}$. The resulting cDNA was stored at $-20^{\circ} \mathrm{C}$.qPCR reaction (40 cycles) at $50{ }^{\circ} \mathrm{C}$ for 2 min, at $95^{\circ} \mathrm{C}$ for $10 \mathrm{~min}$, at $95^{\circ} \mathrm{C} 30$ s, and at $60{ }^{\circ} \mathrm{C}$ for $30 \mathrm{~s}$.After amplificationg,the relative gene expressions were calculated in accordance with the $\Delta \Delta \mathrm{Ct}$ method. Relative miRNA levels were expressed as $2^{-\Delta \Delta C t}$ and ratios to control. Samples were analyzed in triplicates and the data from 6 RT-PCR samples were averaged.

\section{Statistical analysis}

All data were expressed as mean \pm SE. Statistical analysis was performed using IBM SPSS Statistics. The data conformed to normal distribution $(P>0.05)$, and met the homogeneity of variance $(P>0.05)$. Variance analysis was used and LSD was used to compare the two. Data didn't conform to normal distribution $(P<0.05)$, or to normal distribution but didn't met the homogeneity of variance, nonparametric test was used and two comparisons were made, $p<0.05$ was considered statistically significant.

\section{Results}

\section{Protective effect of xenon following LPS and HI injury}

First, we examined the efficiency of LPS and HI in inducing WMD in P3 rat pups as we have done previously[19]. In Group A ,HE staining of brain tissue demonstrated a normal white matter structure and morphology,regular cell arrangement with intact,centrally-positioned nuclei and a clear entoblast at 0,24 , 48 and 72 hours (Fig. 1A-D, respectively). Upon the induction of WMD, HE staining revealed a distorted neural arrangement,loosened cortical structure, distorted nuclear membranes with pyknosis,as well as cellular degeneration and signs of necrosis at $48 \mathrm{~h}$ in group B (Fig. 1E-F). Additionally,we observed an increase in the glial population along with tangled nerve fibers in group B at 72 hours (Fig. 1G-H).The abnormal structure and morphology observed in the white matter following LPS and HI indicate that LPSsensitized HI-induced WMD in group B pups.Following the 3-hour xenon inhalation,the pathological changes induced by LPS and HI were less prominent in group $\mathrm{C}$ compared to group B supporting the notion that xenon could protect against brain injury in premature rats at 0,24,48 and 72 hours(Fig. 1I-L, respectively)

\section{Xenon alleviated the LPS and HI-induced apoptosis}


Next,we performed TUNEL staining to estimate the apoptosis at 24,48 and 72 hours following LPS and $\mathrm{HI}$ treatment.In the group A, TUNEL staining did not reveal significant apoptosis. In contrast, pups in group $B$ showed a significant increase in apoptotic cells in the white matter at all examined time points(Fig. 2A-C). Further,the apoptosis rate was directly correlated with the survival period, i.e. the number of apoptotic cells significantly increased at $48 \mathrm{~h}$ and peaked at $72 \mathrm{~h}$ following LPS and $\mathrm{HI}$ insult (Fig. 2G). Following xenon treatment in group $C$, the number of apoptotic cells significantly decreased at different time points compared to group $B$ which is Xenon untreated $(P<0.05$ at 24,48 and 72 hours, respectively. Figure 2D-F and G).

\section{Xenon up-regulated the expression of miR-210 following white matter injury}

To investigate the effect of xenon treatment on the WMD, we detected the miR-210 level by RT-PCR. Compared with in group $A$, the expression level of miR-210 in neonatal rats' periventricular tissue increased significantly at all time points in group $\mathrm{B}(p<0.05)$. While the expression level of miR-210 in brain tissues of group B was significantly lower at $48 \mathrm{~h}$ and $72 \mathrm{~h}$ than that of group $\mathrm{C}(p<0.05)$, but made no difference with that of group $D$ or group $E(p>0.05)$. Compared with group $C$, the expression of miR210 in brain tissues of group D decreased significantly at $24 \mathrm{~h}$ and $72 \mathrm{~h}(p<0.05)$, and group E decreased significantly at $0 \mathrm{~h}, 24 \mathrm{~h}, 48 \mathrm{~h}$ and $72 \mathrm{~h}(p<0.05)$. And the expression of miR-210 at each group increased firstly and then decreased, and reached the peak at 48 hours.(table 1 and Fig. 3)

Xenon up-regulated the expression of HIF-1a after white matter injury

Next, we investigated the expression of HIF-1a and the efficiency of xenon treatment. Compared to group A, Detection of HIF-1 a protein by Western blot.The expression level of HIF-1 a protein increased firstly and then decreased in each group, reaching peak at $24 \mathrm{~h}$, and was statistically differentiated with each other at every time point $(p<0.05)$. The level of HIF-1 a protein in group B brain tissues was significantly higher than that of group $A$ at each time point,while significantly lower than that of group $C$ and $D$ and $E$ at $0 \mathrm{~h}$, $24 \mathrm{~h}$ and $72 \mathrm{~h}(p<0.05)$. Compared with group $\mathrm{C}$ and group $\mathrm{D}$, the expression of HIF-1 $\mathrm{a}$ in the brain tissue of group E decreased significantly at $24 \mathrm{~h}(p<0.05)$.(table 2 and Fig. 4$)$

\section{Discussion}

The complex pathophysiology of WMD enables multiple targets at different time points of the diseaseprocess. For instance, in the early phase therapies are mainly concentrated on reduction of excitotoxic, oxidative and apoptotic mediators of injury. The development of therapies to reduce brain injury secondary to WMD is important because of the severity of disability that may result.

HIF-1a an important factor in the regulation of hypoxia, and has been proved to be involved in hypoxicischemic preconditioning in multiple species of tissues as a transcription factor. Physiologically, HIF-1a is hydroxylated by prolyl-4-hydroxylases (PHDs) in the oxygen-dependent degradation domain of proline. 
After that,HIF-1a becomes ubiquitin ligase complex, which is degraded by proteasome. HIF-1a is degraded by this mechanism. Under normoxic conditions, HIF-1a protein has a very short half-life (less than 5 min under posthypoxic conditions in cell culture), and decreases in oxygen concentration cause its stability to increase almost immediately, as reduction of PHD activity leadind to degradation of proteasome.[22] HIF-1 a targets a wide variety of genes,including genes involved in energy metabolism,angiogenesis,cell proliferation,and survival.among others,[23]and also plays a role in hypoxic preconditioning in many organs by increasing the expression of HIF-1a,[24, 25]especially in the brain, the role of HIF-1a is well recognized. Prass et al.[26] showed that hypoxia-induced HIF-1a DNA connectivity increased, leading to cerebral ischemic tolerance. Hypoxic stimulation up-regulates the expression of HIF$1 \mathrm{a}$ and EPO protein, produces reactive oxygen species, and plays an obvious neuroprotective role.[27] Recently, a number of microRNAs induced during hypoxia have been identified.One of these microRNAs, miR-210 is strongly induced by HIF-1a and has pleiotropic effects.[28] Fasanaro et al. reported that the expression level of miR-210 in endothelial cells was up-regulated in hypoxic environment, while promoting angiogenesis to a certain extent.[29] It is also closely related to hypoxic-ischemic diseases of the brain. Most studies have shown a direct link between the expression of miR-210 and hypoxia .[30-34] In particular,at the binding site of HIF-1a on its promoter,the expression of miR-210 was significantly upregulated between normal and transformed cells.[33] Some studies have shown that miR-210 plays an important role in cell survival during hypoxia as a highly up-regulated microRNA.,[28, 29]Under normoxic conditions, the presence of miR-210 is not effective, but when hypoxia occurs, the increase of miR-210 expression will reduce the effect of hypoxic environment on cell metabolism to a minimum.[35] miR-210 is a stable target of HIF-1a,that is activated under hypoxic environment, which induces the up-regulation of the expression of miR-210.[17]Hypoxia can promote the expression of HIF-1a increasing at 4 hours, peaking at 8 hours and decreasing at 24 hours,and apoptosis increases significantly at 24 hours after hypoxia,accompanied by down-regulation of HIF-1a expression, that suggesting HIF-1a may play a protective role in regulating apoptosis.[36] Animal experiments and cell culture data showed that the level of miR-210 increased immediately after hypoxic injury, and then decreased for several days. Along with ischemia and hypoxia, the level of HIF-1 $a$ also changed continuously. Therefore, the increase of miR-210 level after $\mathrm{HI}$ injury was regulated by HIF-1a and related to the time of injury.[28, 37] Kelly [38]recently discovered the loop of miR-210 regulating HIF-1 $a$ and identified a new HIF-1a regulatory factor, glycerol-3phosphate dehydrogenase 1-like (GPD1L). The induction of miR-210 by HIF-1a reduced the expression of GPD1L protein, thereby increasing the stability of HIF-1a. In conclusion, HIF-1a protein increased significantly in a certain period of time, peaked at 24 hours, and then gradually decreased to baseline level with the extension of time after $\mathrm{HI}$ injury in neonate rats. The protective effect was enhanced and then weakened. If intervention treatment was given after HI,HIF-1a degradation was inhibited to protect brain tissue,and the expression of miR-210 is related to the level of HIF-1 a protein, that will bring new ideas for the follow-up treatment of neonatal brain damage.Our data suggest that the expression levels of HIF-1 a protein and miR-210 in brain tissue increased after LPS combined with $\mathrm{HI}$ injury(p®0.05), which is consistent with the above study. 
Xenon is an NMDA receptor antagonist that has been precluded from the widespread clinical use as a general anesthetic due to its relatively high cost. $[14,15,39]$ Xenon has well documented neuroprotective properties that were reported in models of premature brain injury, $[7,10,11,39]$ as shown in our previous papers.[19]In this study, we investigated the acute neuroprotective outcomes of xenon in an in vivo model of premature brain injury. In accordance with previous reports, we opted to use a 3-hour xenon treatment interval. $[21,40]$ The present results indicate that xenon has the greatest neuroprotective effect in the range of $37.5-50$ vol\%, which is consistent with previous studies that $50 \%$ xenon can provide sufficient neuroprotective effect .[41-43] On the contrary, others have shown that xenon at $75 \%$ volume concentration has the greatest neuroprotective effect and does not affect oxygenation[41]. However, in addition to neuroprotective effect, xenon at this concentration may have adverse side effects .[43] We choose a mixture of $50 \%$ xenon, $30 \%$ oxygen and $20 \%$ nitrogen to intervene,the mixture has $2.8 \times$ the density but almost the same viscosity as air .[44]In our study, following LPS and HI insult, we observed the expression of miR-210and HIF-1 a elevated,and our results revealed that the administration of $50 \%$ xenon balanced with $30 \%$ oxygen and $20 \%$ nitrogen for 3 hours after injury resulted in a significant increase of miR-210 and HIF-1 $\mathrm{a}(p<0.05)$. These results suggest that xenon can alleviate white matter damage by activating HIF-1 a expression, and the stable expression of HIF-1a can regulate the expression of microRNA-210, which plays a neuroprotective role by inhibiting neuronal apoptosis through related pathways. The available time-window for treatment effectiveness and administration is an important aspect to consider in potential treatments for premature brain injury. Therefore, we investigated the effectiveness of xenon when administered immediately or delayed for 2 or 5 hours following LPS and HIinduced injury. The level of microRNA-210 did not increase significantly, but the level of HIF-1a protein did not decrease significantly.Maintaining and stabilizing the level of HIF-1a protein still served to protect brain tissue. In addition, results revealed that the delay in xenon administration for 5 hours resulted in a significant increase of HIF-1a $(p>0.05)$, which may suggest that xenon still exerts its neuroprotective effects for up to 5 hours after injury, and the regulation between HIF-1a and microRNA-210 is related to the time of injury,and may suggest that xenon still exerts its neuroprotective effects for up to 5 hours after injury,the sooner the beter.

In summary, we showed that xenon is an efficient neuroprotective agent against premature brain injury in a rodent model of WMD. Our results indicated that xenon treatment up-regulated the expression of miR210 and HIF-1 $a$ and improved neurological outcome after WMD. The therapeutic time-window of xenon extends for up to 5 hours. Our findings are in agreement with the previously demonstrated neuroprotective effects of xenon in $\mathrm{HI}$ injury. $[7,19]$ The duration of xenon treatment was relatively short (3 hours) which might suggest that the extension of treatment time might result in better neuroprotection and a longer therapeutic time window. Our findings support the idea that xenon could provide a first-line treatment for white matter injury in premature infants. The development of xenon-closed circuit delivery system and advances in gas extraction technology will potentially lead to enhanced procedures of xenon dosing with reduced cost.[45] Further, xenon is a non-flammable gas that can be easily administered at the bedside. In conclusion, the present study revealed a novel effect of xenon in protection against 
premature brain injury. Future experimental studies and clinical trials would be valuable in providing further insights into xenon neuroprotective efficacy.

\section{Conclusions}

In conclusion, our data indicate that the expression of HIF-1a and miR-210 increased in periventricular tissues and Xe could relieve the white matter damage by up-regulating the expression of HIF-1a and its target gene miR-210.The Xe therapeutic time window was within 5 hours after intervention, the sooner the better.

\section{Abbreviations}

BBB

blood-brain barrier;CCA:Common carotid artery;GPD1L:Glycerol-3-phosphate dehydrogenase 1-like; HE:Hematoxylin and eosin; HI:Hypoxia-ischemia; HIF-1a:hypoxia inducible factor 1a; LPS:lipopolysaccharide; miR-210:microRNA 210; miRNAs:MicroRNAs ; NMDA:N-methyl-D-aspartate ; PHDs:Prolyl-4-hydroxylases; WMD:White matter damage;

Xe

Xenon

\section{Declarations}

\section{Ethics approval and consent to participate}

The protocols, which include all surgical procedures and animal usage, were approved by the Institutional Animal Care and Use Committee of Qingdao University, and conformed to the Guide for the Care and Use of Laboratory Animals by the National Institutes of Health.

\section{Consent for publication}

Not applicable

\section{Availability of data and material}

The data and analysis in this study could be reasonably acquired from the corresponding author.

\section{Competing interests}

None declared

\section{Funding}

Not applicable 


\section{Authors' contributions}

YXY, ZJX,JH had full access to all the data in the study and take responsibility for the integrity of the data and the accuracy of the data analysis. Study concept, design, and drafting of the article:YXY,ZJX,LXH Acquisition, analysis, and interpretation of data. Animal experiment: JJ,XHM, ZLL. All authors have read and approved the manuscript.

\section{Acknowledgements}

Not applicable.

\section{References}

1. Peterson BS, Vohr B, Staib LH, Cannistraci CJ, Dolberg A, Schneider KC, et al. Regional brain volume abnormalities and long-term cognitive outcome in preterm infants. Jama. 2000;284(15):1939-47.

2. Miller SP, Ferriero DM, Leonard, Piecuch R, Glidden DV, Partridge JC, et al. Early brain injury in premature newborns detected with magnetic resonance imaging is associated with adverse early neurodevelopmental outcome. Pediatr. 2005;147(5):609-16.

3. Dammann O, Leviton A. Inflammation, brain damage and visual dysfunction in preterm infants. Semin Fetal Neonatal Med. 2006;11(5):363-8.

4. Khwaja O, Volpe JJ. Pathogenesis of cerebral white matter injury of prematurity. Archives of disease in childhood Fetal neonatal edition. 2008;93(2):F153-61.

5. Liu W, Liu Y, Chen H, Liu K, Tao HY,Sun XJ. Xenon preconditioning: molecular mechanisms and biological effects. Medical gas research. 2016;3(1):3.

6. Ma D, Hossain M, Pettet GK, Luo Y, Lim T, Akimov S,et al. Xenon preconditioning reduces brain damage from neonatal asphyxia in rats. Journal of cerebral blood flow metabolism: official journal of the International Society of Cerebral Blood Flow Metabolism. 2006;26(2):199-208.

7. Yang T, Zhuang L, Rei Fidalgo AM, Petrides E, Terrando N, Wu X, et al. Xenon and sevoflurane provide analgesia during labor and fetal brain protection in a perinatal rat model of hypoxia-ischemia. PloS one. 2012;7(5):e37020.

8. Arola OJ, Laitio RM, Roine RO, Gronlund J, Saraste A, Pietila M,et al. Feasibility and cardiac safety of inhaled xenon in combination with therapeutic hypothermia following out-of-hospital cardiac arrest. Critical care medicine. 2013;41(9):2116-24.

9. Azzopardi D, Robertson NJ, Kapetanakis A, Griffiths J, Rennie JM, Mathieson SR, et al. Anticonvulsant effect of xenon on neonatal asphyxial seizures. Archives of disease in childhood Fetal neonatal edition. 2013;98(5):F437-9.

10. Sheng SP, Lei B, James ML, Lascola CD, Venkatraman TN, Jung JY,et al. Xenon neuroprotection in experimental stroke: interactions with hypothermia and intracerebral hemorrhage. Anesthesiology. 2012;117(6):1262-75. 
11. Bickler PE, Warren DE, Clark JP, Gabatto P, Gregersen M, Brosnan H. Anesthetic protection of neurons injured by hypothermia and rewarming: roles of intracellular $\mathrm{Ca} 2+$ and excitotoxicity. Anesthesiology. 2012;117(2):280-92.

12. Goto T, Nakata Y, Morita S. Will xenon be a stranger or a friend?: the cost, benefit, and future of xenon anesthesia. Anesthesiology. 2003;98(1):1-2.

13. Rossaint R, Reyle-Hahn M, Schulte Am Esch J, Scholz J, Scherpereel P, Vallet B,et al. Multicenter randomized comparison of the efficacy and safety of xenon and isoflurane in patients undergoing elective surgery. Anesthesiology. 2003;98(1):6-13.

14. Neukirchen M, Hipp J, Schaefer MS, Brandenburger T, Bauer I, Winterhalter M,et al. Cardiovascular stability and unchanged muscle sympathetic activity during xenon anaesthesia: role of norepinephrine uptake inhibition. Br J Anaesth. 2012;109(6):887-96.

15. Armstrong SP, Banks PJ, McKitrick TJ, Geldart CH, Edge CJ, Babla R,et al. Identification of two mutations (F758W and F758Y) in the N-methyl-D-aspartate receptor glycine-binding site that selectively prevent competitive inhibition by xenon without affecting glycine binding. Anesthesiology. 2012;117(1):38-47.

16. Dickinson R, Peterson BK, Banks P, Simillis C, Martin JC, Valenzuela CA, et al. Competitive inhibition at the glycine site of the $\mathrm{N}$-methyl-D-aspartate receptor by the anesthetics xenon and isoflurane: evidence from molecular modeling and electrophysiology. Anesthesiology. 2007;107(5):756-67.

17. Ma D, Lim T, Xu J, Tang H, Wan y, Zhao H, et al. Xenon preconditioning protects against renal ischemic-reperfusion injury via HIF-1alpha activation. J Am Soc Nephrol. 2009;20:713-20.

18. CampsC,Buffa FM, Colella S, Moore J, Sotiriou C, Sheldon H,et al. hsa-miR-210 Is induced by hypoxia and is an independent prognostic factor in breast cancer. Clin Cancer Res. 2008;14(5):1340-8.

19. Xiangyun Yin J, Zhao HJiang,LiangliangLi,Jian, Jiang,Hongmin XI. Impact of Xenon on CLIC4 and Bcl-2 Expression in Lipopolysaccharide and Hypoxia-Ischemia-Induced Periventricular White Matter Damage.Neonatology 2018;113:339-346.

20. Wang LW, Chang YC, Chen SJ, Tseng CH, Tu YF, Liao NS,et al. TNFR1-JNK signaling is the shared pathway of neuroinflammation and neurovascular damage after LPS-sensitized hypoxic-ischemic injury in the immature brain. J Neuroinflamm. 2014a;11:215.

21. Back SA, Han BH, Luo NL, Chricton CA, Xanthoudakis S, Tam J, et al. Selective vulnerability of late oligodendrocyte progenitors to hypoxia-ischemia. The Journal of neuroscience: the official journal of the Society for Neuroscience. 2002;22(2):455-63.

22. RishiM T, Selvaraju V,Thirunavukkarasu M, Shaikh IA,Takeda K, Fonq GH,et al. Deletion of prolyl hydroxylase domain proteins (PHD1, PHD3) stabilizes hypoxia inducible factor-1 alpha, promotes neovascularization, and improves perfusion in a murine model of hind-limb ischemia. Microvasc Res. 2015;97:181-8.

23. Semenza GL. Targeting HIF-1 for cancer therapy. Nat Rev Cancer. 2003;3:721-32.

24. ShizukudaY MalletRT, Downey LeeSC. HF. Hypoxic preconditioning of ischaemic canine myocardium. Cardiovasc Res. 1992;26:534-42. 
25. CaiZ,Manalo DJ, Wei G, Rodriquez ER, Fox-Talbot K, Lu H,et al. Hearts from rodents exposed to intermittent hypoxia or erythropoietin are protected against ischemia-reperfusion injury. Circulation. 2003;108(1):79-85.

26. Prass K, Scharff A, Ruscher K, Lowl D, Muselmann C, Victorov l,et al. Hypoxia-induced stroke tolerance in the mouse is mediated by erythropoietin. Stroke. 2003;34(8):1981-6.

27. LiuJ, Narasimhan P, Yu F, Chan PH. Neuroprotection by hypoxic preconditioning involves oxidative stress-mediated expression of hypoxia-inducible factor and erythropoietin. Stroke. 2005;36:1264-9.

28. Kulshreshtha R, Ferracin M, Wojcik SE, Garzon R, Alder H, Agosto-Perez FJ, et al. A microRNA signature of hypoxia. Mol CellBiol. 2007;27:1859-67.

29. FasanaroP,Alessandra Y, Di Stefano V, Melchionna R, Romani S, Pompilio G, et al. MicroRNA-210 modulates endothelial cell response to hypoxia and inhibits the receptor tyrosine kinase ligand Ephrin-A3. Biol Chem. 2008;283(23):15878-83.

30. HuangX. LeQT, GiacciaAJ. MiR-210-micromanager of the hypoxia pathway. Trends Mol Med. 2010;16:230-7.

31. Ivan M,HuangX. miR-210: fine-tuning the hypoxic response. Adv Exp Med Biol. 2014;772:205-27.

32. QinQ FW, Baosheng L. Multiple functions of hypoxia-regulated miR-210 in cancer. J Exp Clin Cancer Res. 2014;33:50.

33. Malm PulkkinenK, Turunen T, Koistinaho M, Yia-Herttuala J. S. Hypoxia induces microRNA miR-210 in vitro and in vivo ephrin-A3 and neuronal pentraxin 1 are potentially regulated by miR-210. FEBS Lett. 2008;582:2397-401.

34. Kim HW, Haider HK, Jiang S, Ashraf M. Ischemic preconditioning augments survival of stem cells via miR-210 expression by targeting caspase-8-associated protein 2. J Biol Chem. 2009;284:33161-8.

35. ChanSY,Zhang YY, Hemann C, Mahoney CE, Zweier JL, Loscalzo J. MicroRNA-210 controls mitochondrial metabolism during hypoxia by repressing the iron-sulfur cluster assembly proteins ISCU1/2[J]. Cell Metab. 2009;10(4):273-84.

36. LiL,Qu Y, Li J, Xiong Y, Mao M, Mu D. Relationship between HIF-1alpha expression and neuronal apoptosis in neonatal rats with hypoxia-ischemia brain injury. Brain Res. 2007;1180:133-9.

37. ZengL,Liu J, Wang Y, Wang L,WengS, Tang Y,et al. MicroRNA-210 as a novel blood biomarker in acute cerebral ischemia. Front Biosci (Elite Ed). 2011;3:1265-72.

38. KellyTJ SouzaAL, ClishCB,Puiqserver P. A hypoxia-induced positive feedback loop promotes hypoxiainducible factor 1 alpha stability through miR-210 suppression of glycerol-3-phosphate dehydrogenase 1-like. Mol Cell Biol. 2011;31:2696-706.

39. Banks P, Franks NP, Dickinson R. Competitive inhibition at the glycine site of the N-methyl-D-aspartate receptor mediates xenon neuroprotection against hypoxia-ischemia. Anesthesiology. 2010;112(3):614-22.

40. Campos-Pires R, Armstrong SP, Sebastiani A, Luh C, Gruss M, Radyushkin K,et al. Xenon improves neurologic outcome and reduces secondary injury following trauma in an in vivo model of traumatic 
brain injury. Critical care medicine. 2015;43(1):149-58.

41. Ma D, Hossain M, Chow A, Ashad M, Battson RM, Sanders RD,et al. Xenon and hypothermia combine to provide neuroprotection from neonatal asphyxia. Ann Neurol. 2005;58,(2):182-93.

42. Dingley J, Tooley J, Porter $\mathrm{H}$, Thoresen M. Xenon provides short-term neuroprotection in neonatal rats when administered after hypoxia-ischemia. Stroke. 2006;37:501-6.

43. Abraini JH, David HN, Lemaire M. Potentially neuroprotective and therapeutic properties of nitrous oxide and xenon. Ann N Y Acad Sci. 2005;1053:289-300.

44. Jaffrin MY. KesicP.Airway resistance: a fluid mechanical approach. Appl Physiol. 1974;36:354-61.

45. Faulkner SD, Downie NA, Mercer CJ, Kerr SA, Sanders RD, Robertson NJ. A xenon recirculating ventilator for the newborn piglet: developing clinical applications of xenon for neonates. Eur $\mathrm{J}$ Anaesthesiol. 2012;29(12):577-85.

\section{Tables}

Due to technical limitations, table $1 \& 2$ is only available as a download in the Supplemental Files section."

\section{Figures}
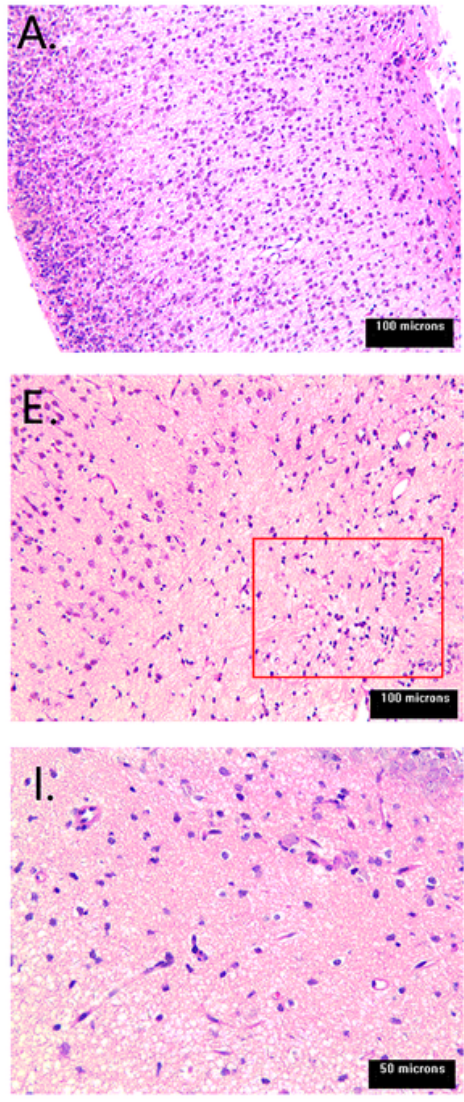
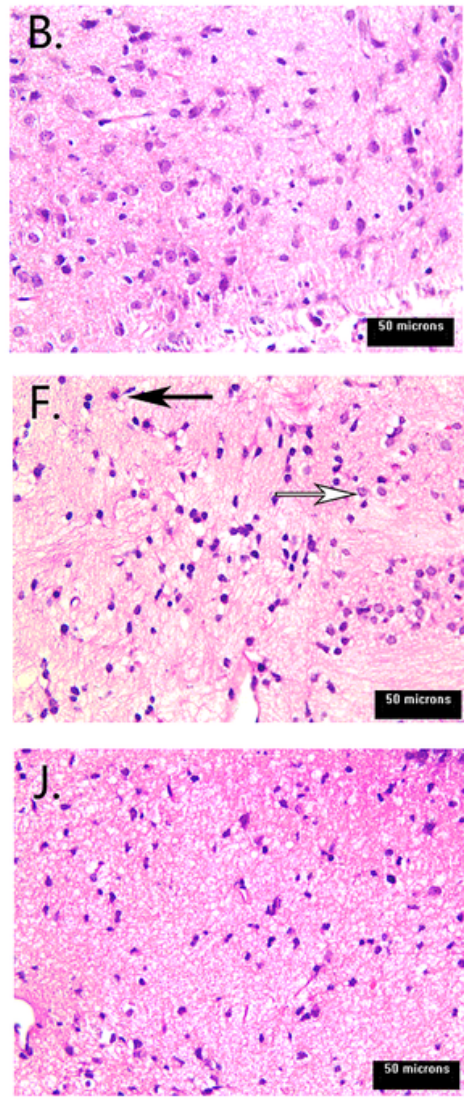
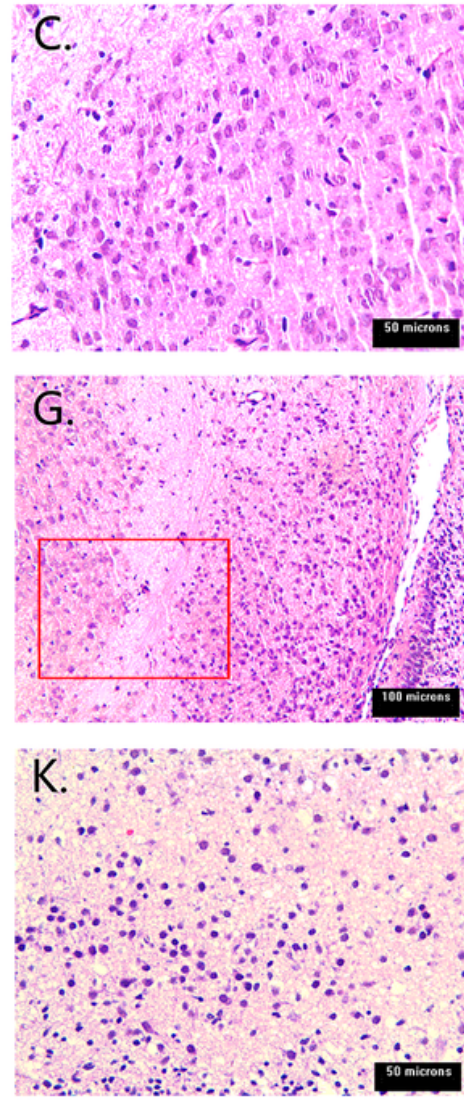
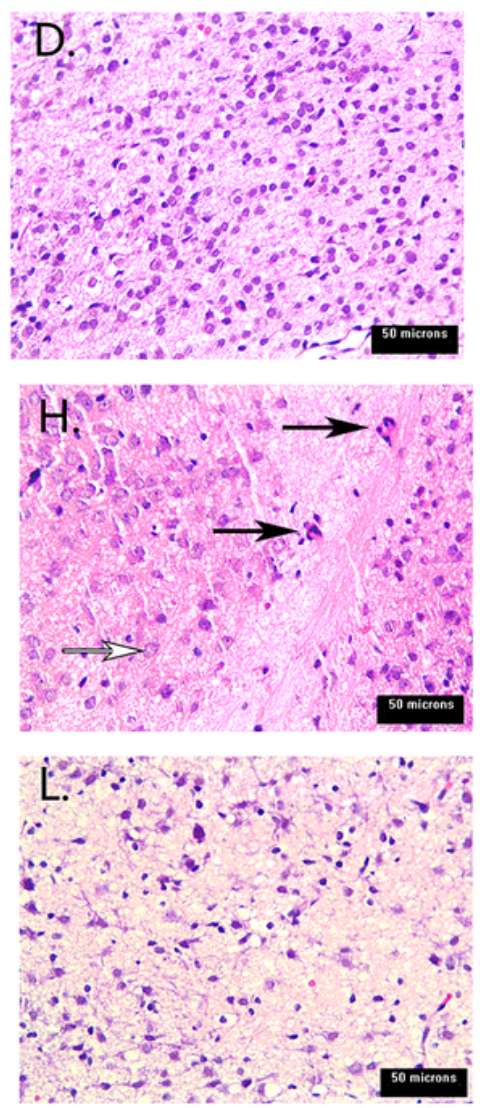

Figure 1 
Representative hematoxylin and eosin-stained coronal brain sections from postnatal day 3 SD rat pups. (A-D ) Normal cortical and white matter structure in the sham-operated pups (group A) at 0, 24, 48 and 72 hours, respectively $(n=6) .(E-H)$ Distorted cytoarchitecture, nuclear pyknosis (black arrows) observed in the brain sections following LPS and $\mathrm{HI}$ in group $B$ at 48 and 72 hours, $(n=6)$. ( $(-L)$ Protective effect of xenon in group $C$ pups at $0,24,48$ and 72 hours, $(n=6)$. Scale bar=50 $\mu \mathrm{m}$ inB-D, F,H-L; Scale bar=100 $\mu \mathrm{m}$ in A,E andG.
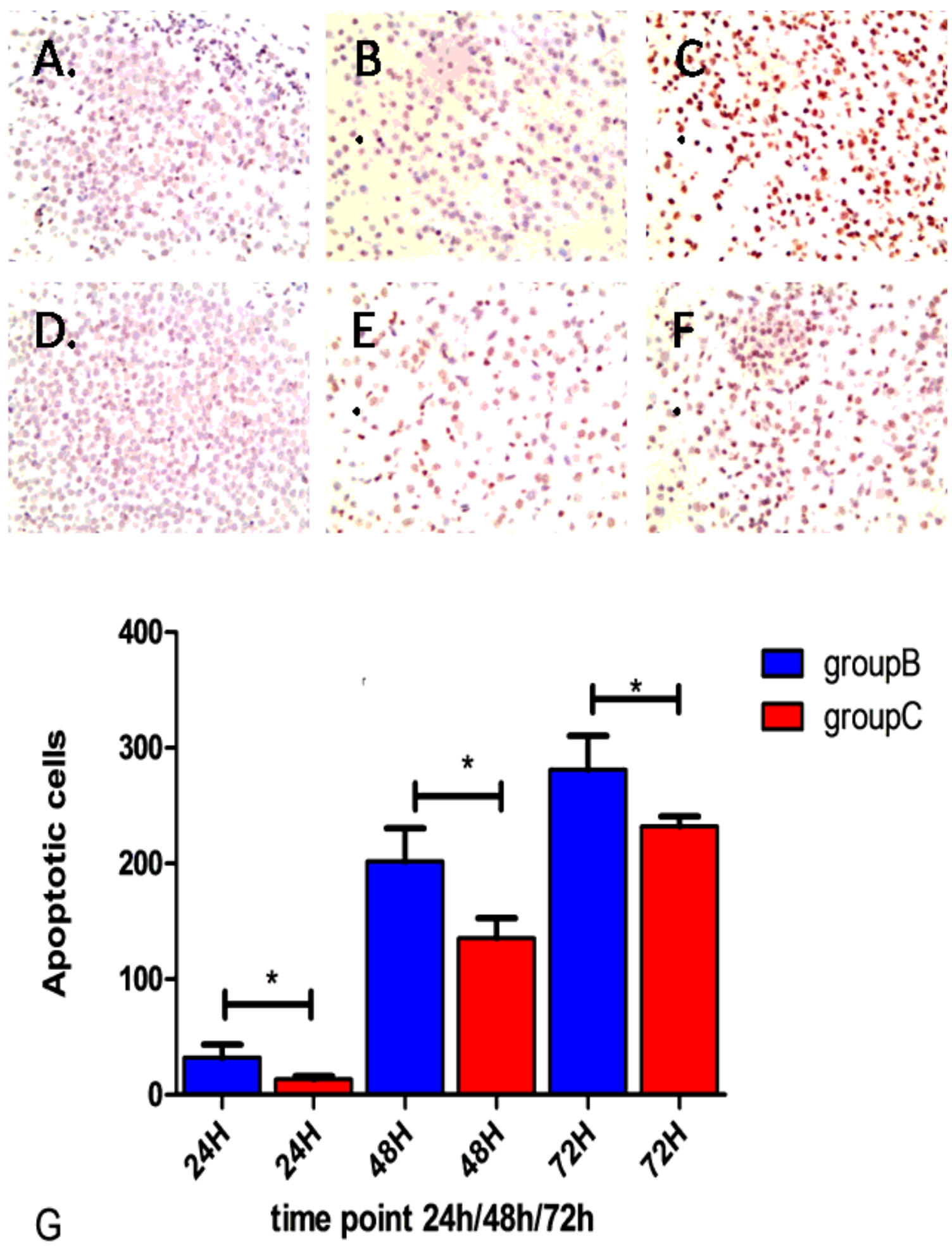

Figure 2 
Representative images of TUNEL staining from postnatal day 3 SD rat pups. (A-C)Following LPS and HI the number of apoptotic cells increased significantly in group B pups at 24,48 and 72 hours (n=3). (DF)Protective effect of xenon in group $C$ pups at 24,48 and 72 hours, $(n=3) . \otimes G \otimes B$ ar chart representing the average number of TUNEL positive cells of group $B$ and groupC, ${ }^{*}<0.05$ compared to group $B$.

a

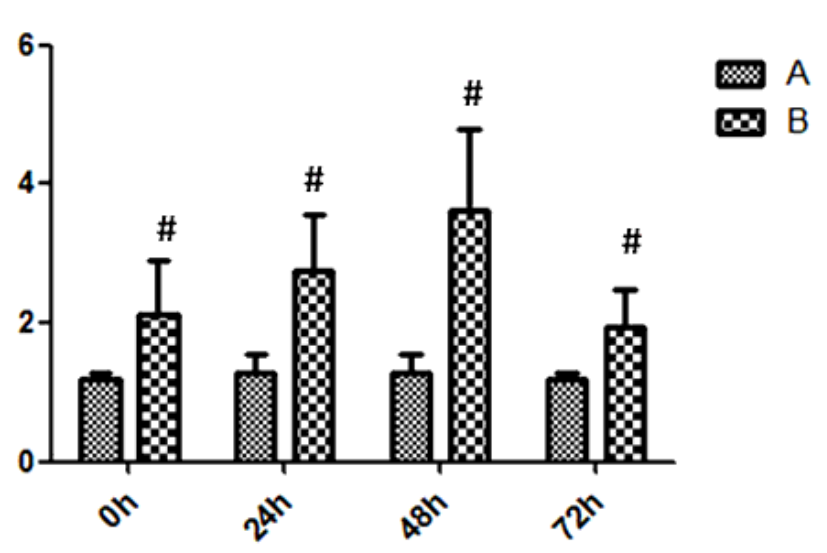

b

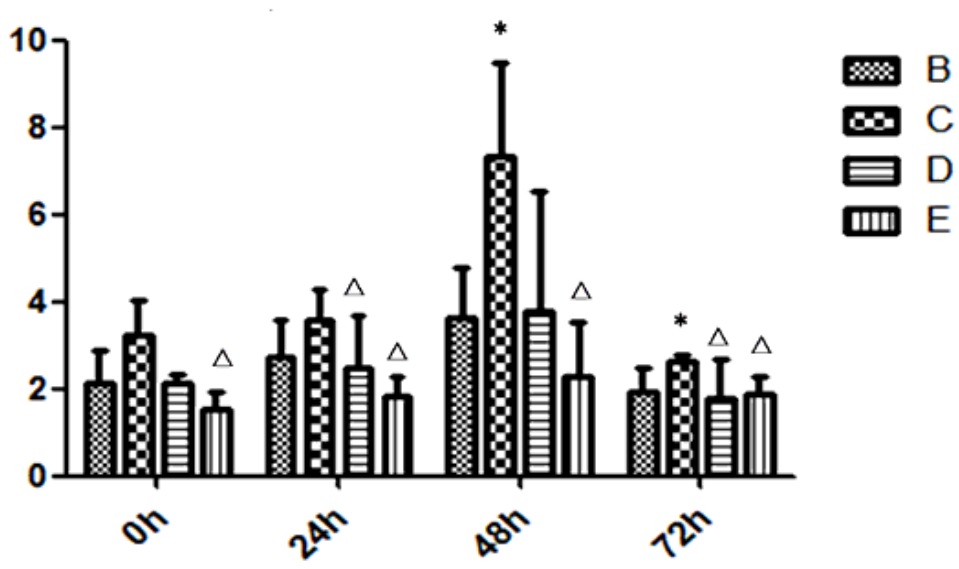

Figure 3

Expression of miR-210 in the brain tissue at different time points in group A-E a: Bar charts representing the relative expression of miR-210 at $0,24,48$ and 72 hours in group $A$ and $B, \# p<0.05$ compared to group $A(n=6)$. b: Bar charts representing the relative expression of miR-210 in groups $B, C, D$ and $E$ at 0 , 24,48 and 72 hours. * $p<0.05$ compared to group $B(n=6), \triangle p<0.05$ compared to group $C(n=6)$

a

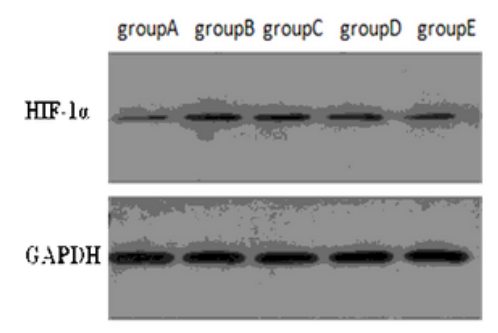

c

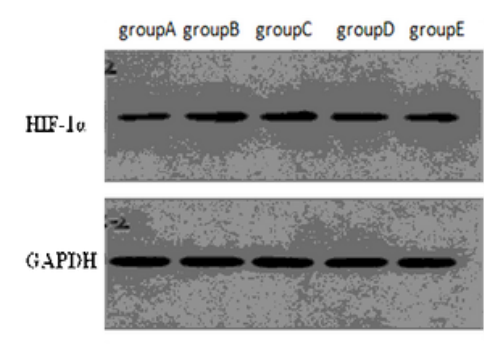

b
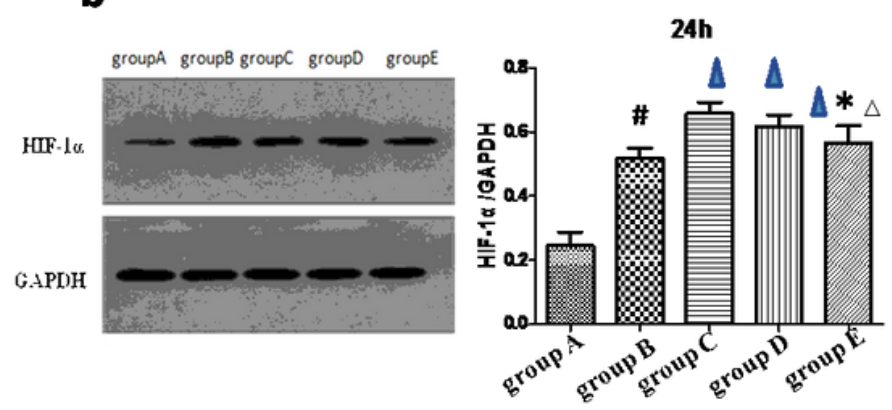

d

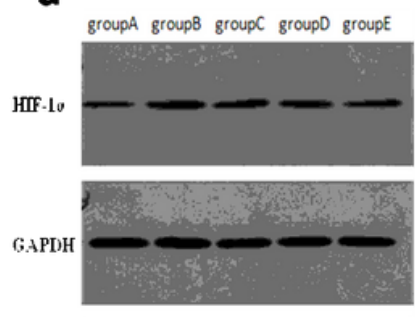

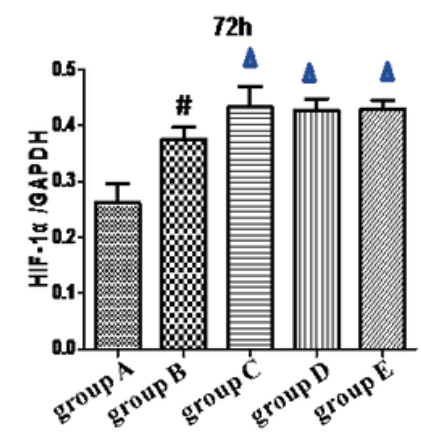




\section{Figure 4}

The expression of HIF-1a in the brain tissue at different time points in groups A -E a Western blot analysis of HIF-1 a expression at $0 \mathrm{~h}$ among the different groups, and bar chart representing the quantitative analysis of HIF-1a expression level normalized to GAPDH level, $\# p<0.05$ compared to group $A, \boldsymbol{\Delta} p<$ 0.05 compared to group $B(n=6)$. b Western blot analysis of HIF-1 a expression among the different groups at $24 \mathrm{~h}$, and bar chart representing the quantitative analysis of HIF-1 a expression level normalized to GAPDH level, $\# p<0.05$ compared to group $A \Delta p<0.05$ compared to group $B$, ${ }^{\star} p<0.05$ compared to group $C, \triangle p<0.05$ compared to group $D(n=6)$. c HIF-1a expression among the different groups at $48 \mathrm{~h}$, and bar chart representing the quantitative analysis of HIF-1 a expression level normalized to GAPDH level, $\# p<0.05$ compared to group $A, \Delta p<0.05$ compared to group $B(n=6)$.d HIF-1a expression among the different groups at $72 \mathrm{~h}$, and bar chart representing the quantitative analysis of HIF-1a expression level normalized to GAPDH level, \# $p<0.05$ compared to group $A, \boldsymbol{\Delta} p<0.05$ compared to group $B(n=6)$.

\section{Supplementary Files}

This is a list of supplementary files associated with this preprint. Click to download.

- table12.pdf

- table12.pdf 